\title{
ONDES DE SURFACE AU-DESSUS D'UN FOND CONSTITUE DE RIDES
}

\author{
J. Brossard, F.Marin, M.Belorgey \\ Laboratoire de Mecanique des Fuides \\ Université du Havre, 25 rue Philippe Lebon, B.P. 540, 76058 LE HAVRE CEDEX
}

\begin{abstract}
SURFACE WAVES OVER RIPPLED BED. The flow generated in the neighbourhood of periodical ripples distributed on a wave flume bottom has been experimentally investigated. These ripples are asymmetric and have sharp crests. Vortices evolutions generated at each ripple crest,are analysed thanks to the rotational field. Results exhibit a non-symetrical evolution of opposite sign rotational structures. The stationary component of velocity flow field may explain the stability of ripple systems on sea beds.
\end{abstract}

\section{1-INTRODUCTION}

L'intérêt de l'étude des rides se formant sur les côtes sableuses sous l'action des vagues n'est plus à démontrer. Les rides sont le résultat du transport sédimentaire à proximité du fond et participent à la dissipation d'énergie sur les fonds et à la remise en suspension des sédiments.

Nos travaux expérimentaux portent sur la description quantitative des champs de vitesse. L'écoulement est celui induit par la propagation d'une onde de surface sensiblement monochromatique dans un canal à houle dont le fond, rigide, est constitué de rides anguleuses et asymétriques. L'écoulement ambiant au voisinage du fond est faiblement asymétrique. Le nombre de KeuleganCarpenter $\alpha=k A$ est de 5,8 , le facteur de pente $\varepsilon=k a$ de 0,42 et le nombre de Reynolds $\mathrm{R}=\mathrm{aU} U_{0} / v$ de 81 , où $\mathrm{k}$ est le nombre d'onde des rides, $\mathrm{A}$ l'amplitude d'oscillation de l'écoulement ambiant, 2 a la hauteur des rides et $U_{0}$ l'amplitude de ìa vitesse de l'écoulement ambiant. 


\section{2-MESURES ET RESULTATS}

Les conditions expérimentales sont précisées sur la figure 1:

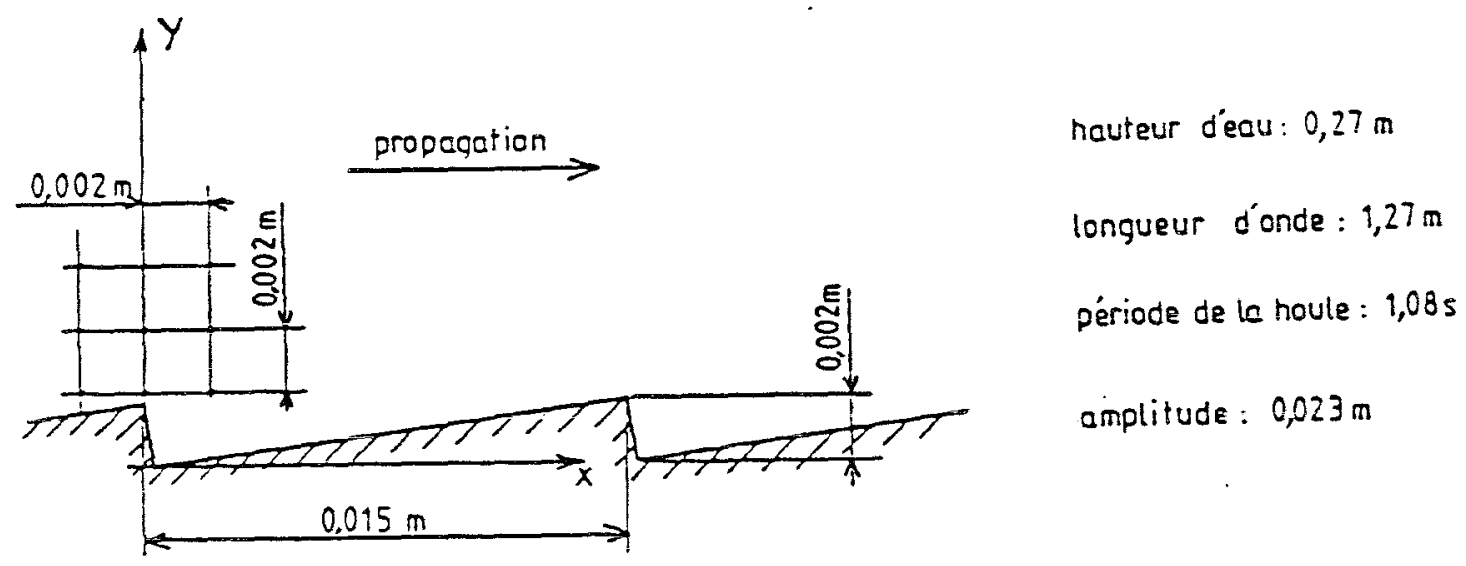

fig. 1 : schema des conditions experimentales

Actuellement, la vélocimétrie laser permet la mesure des vitesses instantanées dans un écoulement sans perturber celui-ci. Cest cette technique qui est exploitée pour analyser les phénomènes liés à la houle; nous travaillons avec un vélocimètre deux composantes en diffusion avant. En chaque point de mesure, on effectue une acquisition des vitesses sur une dizaine de périodes et, par la détection d'un front de la déformation de la surface libre, nous pouvons caler en phase chaque acquisition. On effectue ensuite une partition de chaque échantillon en autant de parties qu'il y a de périodes puis, chaque partie est découpée en cinquante intervalles. Sur chacun de ces intervalles nous calculons entre autres une valeur moyenne des deux composantes de la vitesse. Nous reconstituons alors une période moyenne en calculant la moyenne sur le nombre de périodes des valeurs de vitesse; et ce pour chacun des cinquante intervalles. Ainsi nous pouvons représenter les champs de vitesse "instantanée" avec une résolution temporelle égale à un cinquantième de la période de la houle. $\mathrm{La}$ résolution spatiale dans le cas de l'expérimentation présentée ici est de $0.002 \times 0.002 \mathrm{mxm}$.

Pour décrire l'évolution des tourbillons au voisinage des rides, nous avons calculé en chaque point du champ, la composante transversale du rotationnel des vitesses. Les structures tourbillonnaires sont ainsi caractérisées par les zones de rotationnel. 
Nous présentons, figure 2 , les champs de vitesse et de rotationnel lissés par la méthode des moindres carrés à dix instants régulièrement répartis sur la période. Les modules des rotationnels sont proportionnels aux rayons des ares de cercle. Le fait de visualiser les vecteurs vitesse simultanément avec les rotationnels permet de mieux comprendre le déplacement des tourbillons. L'instant $\omega t=0$ correspond au passage du sommet de la vague en $x=0$. Seule la moitié inférieure du domaine de mesure a été représentée sur la figure. De plus, le mode de calcul des dérivées partielles nous oblige à éliminer les valeurs de rotationnel sur le pourtour de la zone de mesure.

\section{3-ANALYSE DES RESULTATS}

Pour suivre l'évolution des structures tourbillonnaires, nous faisons l'hypothèse que les phénomènes apparaissant à proximité des rides ont une périodicité spatiale correspondant à la longueur d'onde des rides.

La forme des rides induit la génération de deux structures tourbillonnaires à partir des crêtes. L'une, négative (tournant dans le sens orbital de la houle) se forme en aval de la crête et l'autre, positive, en amont.

La structure négative apparaît clairement à partir de la phase $\omega t=0,63$; mais il semble que sa formation ait commencé bien avant, au creux de la ride (hors de la zone de mesure). Le tourbillon s'intensifie alors de plus en plus, ceci jusqu'à la phase $\omega t=1,26$ où le rotationnel est supérieur à $80 \mathrm{rd} / \mathrm{s}$ en module. Sa convection vers l'amont commence à la phase $\omega t=1,51$ correspondant à l'inversion du sens de l'écoulement join des rides. A la phase $\omega t=3,14$ il a parcouru une longueur de ride et passe au-dessus de la structure positive en cours de formation. A la phase $\omega t=4,40$, il est au maximum de sa course vers l'amont. A partir de la phase $\omega t=4,65$ son intensité décroît et il s'élève sensiblement en étant convecté vers l'aval. Au-delà de $\omega t=6,91$, il devient très difficile de le suivre. Le déplacement de la structure négative est donc en phase avec l'écoulement ambiant. Il est, durant la période suivant sa formation, d'environ deux fois la longueur d'onde $L$ des rides vers l'amont et sans doute d'une distance voisine vers l'aval. Il faut noter que l'amplitude des déplacements des particules dans l'écoulement ambiant (à $y=20,2 \mathrm{~mm}$ ) est d'environ 0,9L. L'élévation peut être estimée à environ $0,3 \mathrm{~L}$.

La structure tourbillonnaire positive se formant à l'amont de la crête apparaît à partir de la phase $\omega t=2,51$; son intensité croît jusque vers $\omega t=4,40$; le rotationnel s'élève alors à plus de $80 \mathrm{rd} / \mathrm{s}$ et elle est ensuite convectée vers l'aval 
jusqu'à l'inversion de l'écoulement à la phase $\omega t=1,51$. Son déplacement est alors d'environ 2L. Ensuite son intensité décroît beaucoup et il devient difficile de décrire son comportement. Son élévation est beaucoup plus faible que celle de la structure négative. Celà peut être dû à la dissymétrie des rides, mais cela reste à confirmer.

fig. 2a : Vitesses et composantes transversales du rotationnel

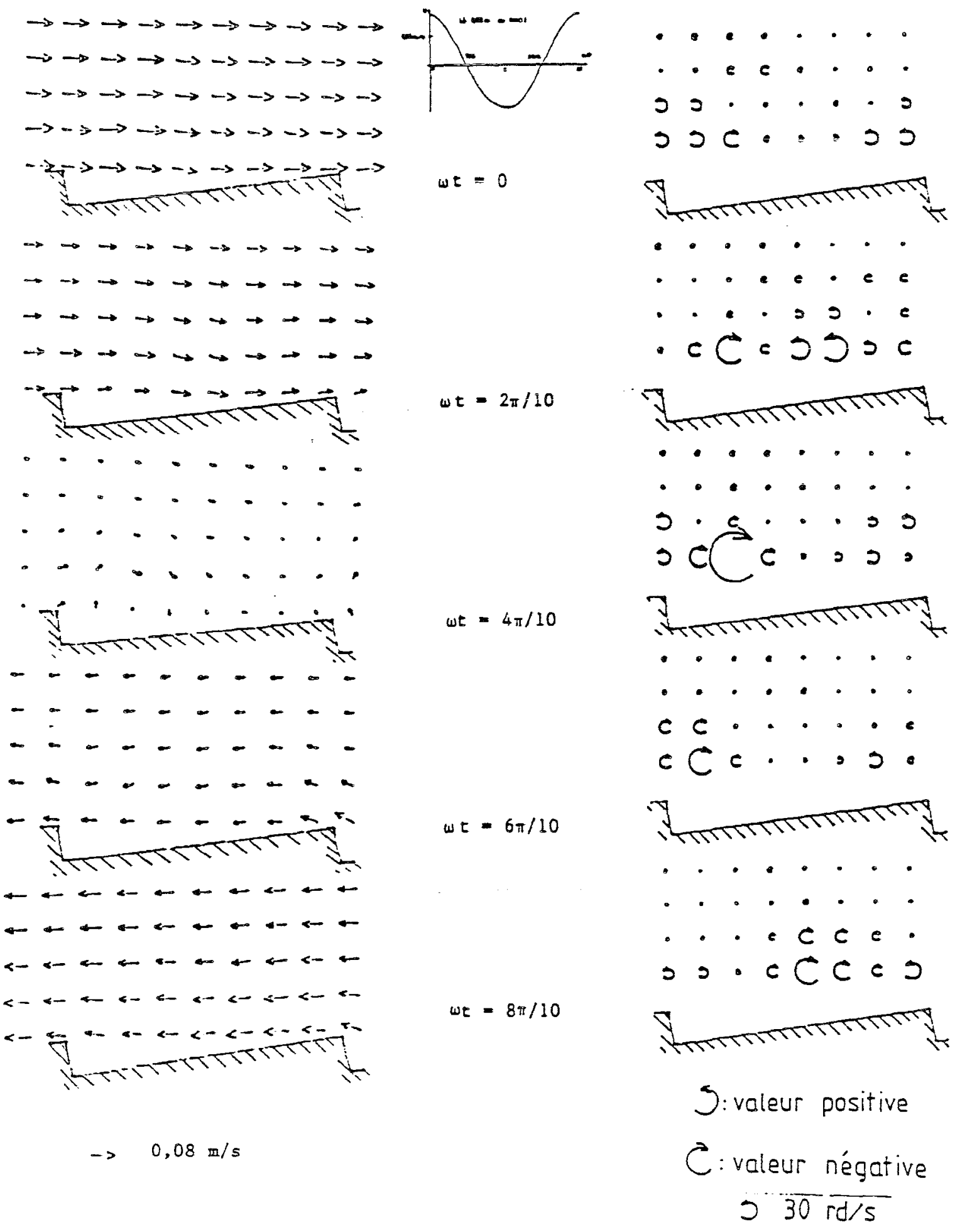


fig. $2 b$ : Vitesses et composantes transversales du rotationnel

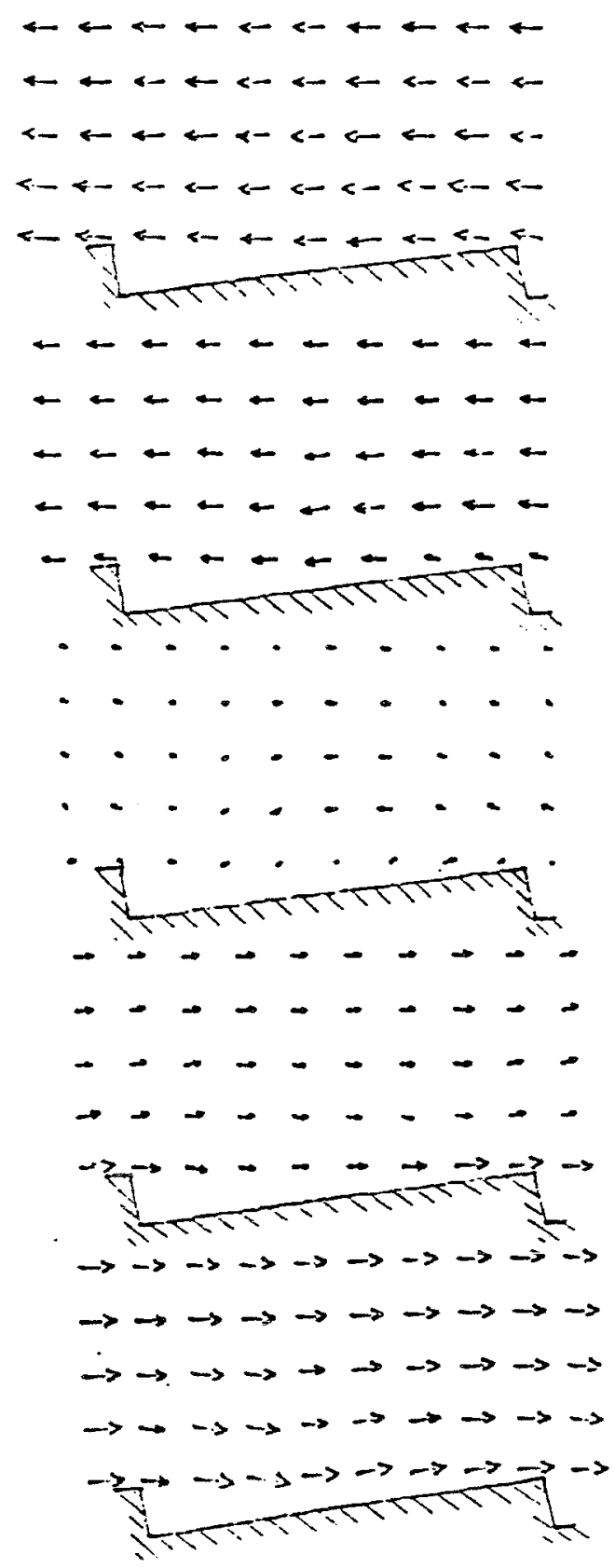

$\omega t=10 \pi / 10$

$\omega t=12 \pi / 10$

$\omega t=14 \pi / 10$

$\omega t=16 \pi / 10$

$\omega t=18 \pi / 10$

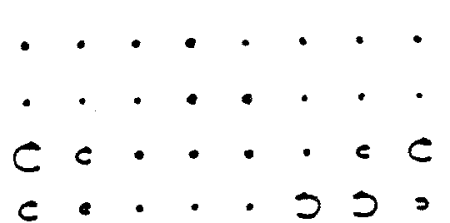

iु

-..$\cdot \cdot \cdot$

. . •. . . .

- $e c c . \cdot \cdot$

$S, \cdot c \cdot 350$

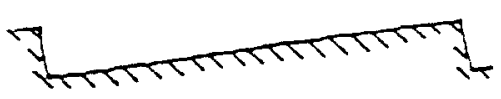

- $\bullet \cdot \cdot$

- $c c c \dot{j} \dot{j} \dot{j}$
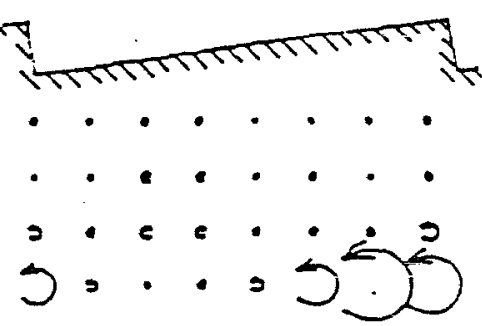

भाकासा

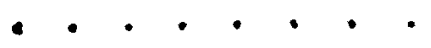

. . . e. . .

c $c 2$ c c c

$c, 50$.

'ु 


\section{4-TOURBILLONS ET TRANSPORT SEDIMENTAIRE.}

En examinant les champs de rotationnel on comprend bien que les structures tourbillonnaires puissent "arracher" des particules des fonds sableux. On conçoit aussi qu'une partie de ce matériau sédimentaire soit transportée par les tourbillons sur des distances assez grandes (de l'ordre de $2 \mathrm{~L}$ horizontalement et $0,5 \mathrm{~L}$ verticalement) au moins pour les sédiments les moins denses.

On peut remarquer aussi, que les tourbillons ont des temps de formation assez long, voisins d'une demie-période. Pendant leur genèse, ces tourbillons restent à peu près stationnaires et l'on peùt penser que cela a un effet sur le transport des sédiments les plus denses.

Cela est plus clairement mis eñ évidence si on représente la composante stationnaire des champs de vitesses obtenues en moyennant les vitesses sur une période. La figure 3 présente cette composante stationnaire.

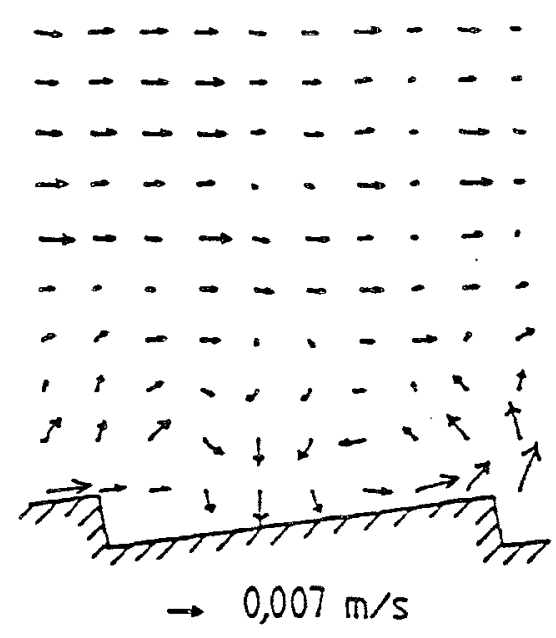

fig. 3 : schéma de la composante stationnaire

Plusieurs remarques peuvent être faites. La première est que l'écoulement est "asymétrique". A $20 \mathrm{~mm}$ du fond, la composante stationnaire de vitesse est dans le sens de propagation et a une intensité environ quinze fois plus faible que l'amplitude de la vitesse au même endroit. Cela implique un transport dans le sens de propagation, fait généralement constaté dans toutes nos études.

La deuxième remarque est qu'il existe deux cellules tourbillonnaires qui traduisent les longs temps de formation et de présence des tourbillons engendrés aux crêtes. Il faut préciser ici , que l'absence de mesure au creux des rides, ne 
permet pas d'observer l'enroulement des lignes de champ de la cellule "négative" immédiatement à l'aval de la crête.

La présence de ces cellules peut permettre de comprendre le maintien des systèmes de rides sur les fonds marins en expliquant la migration de particules des creux de rides vers les crêtes. Ces cellules ont une ampleur verticale importante et leur influence s'exerce vers le haut jusqu'à plus qu'une demielongueur d'onde de ride.

\section{5-CONCLUSION}

La présence de rides sur les fonds marins provoque la formation de structures tourbillonnaires en phase avec l'écoulement environnant. Ces structures se formant aux crêtes, peuvent provoquer une remise en suspension de sédiments (les moins denses) loin au-dessus des rides qui semblent être entretenues par des cellules de circulation stationnaires.

Nous remercions la Direction des Recherches Eudes et Techniques pour le soutien apporté à ces travaux.

\section{REFERENCES}

R.A. BAGNOLD, Motion of waves in shallow water Interaction between waves and sand bottoms, Proc. Roy. Soc. A 187, 1946, p.1-15.

M.R. CARSTENS, F.M. NEILSON et M.D. ALTINBILEK, Bed forms generated in the laboratory under oscillatory flow, Coastal Engng Res. Center, Tech. Memo n²8, 1969.

T. HARA et C.C. MEI, Oscillating flows over periodic rippies, J. Fluid Mech., 211, 1990, p. 183-209.

A.K.F.M. HUSSAIN, Coherent structures, reality and myth, Phys. Fluids, 26 (10), 1983, p. 2816-2850.

K.E.B. LOFQUIST, Sand ripple growth in an oscillatory flow water tunnel, Coastal Engng Res. Center, Tech Paper, n 78-5, 1978.

M.S. LONGUET-HIGGINS, Oscillating flow over steep sand ripples, J. Fluid Mech., 107, 1981, p. 1-35.

S.SATO, N. MIMURA et A. WATANABE, Oscillatory boundary layer flow over rippled beds, Proc. 19th Conf. on Coastal Engng, 1984, p. 22932309.

J.F.A. SLEATH, A contribution to the study of vortex ripples, J. Hydraul. Res., 13, 1975, p. 315-328. 